Acta Crystallographica Section D

Biological

Crystallography

ISSN 0907-4449

${ }^{a}$ Institute of Biological Chemistry, Academia Sinica, Taipei, Taiwan, and ${ }^{\mathbf{b}}$ Institute of Biochemical Sciences, National Taiwan University, Taipei, Taiwan

t These authors contributed equaly in the supervision of this paper.

Correspondence e-mail: ahjwang@gate.sinica.edu.tw

\section{The 1.35 ^ structure of cadmium-substituted TM-3, a snake-venom metalloproteinase from Taiwan habu: elucidation of a TNFa-converting enzyme-like active-site structure with a distorted octahedral geometry of cadmium}

The crystal structure of TM-3, a small snake-venom metalloproteinase (SVMP) isolated from Taiwan habu (Trimeresurus mucrosquamatus), was determined at $1.35 \AA$ resolution with resultant $R$ and $R_{\text {free }}$ values of 0.181 and 0.204 , respectively. The overall structure of TM-3 is an oblate ellipsoid that contains three disulfide crosslinks, Cys118-Cys197, Cys159Cys181 and Cys161-Cys164. It exhibits the typical structural features of SVMPs and is closely related to the structure of the catalytic proteinase domain of TNF $\alpha$-converting enzyme (TACE). In the present structure, the essential catalytic zinc ion was found to be replaced by a cadmium ion during crystallization, as revealed by atomic absorption analysis and $\mathrm{X}$-ray data. This cadmium ion is bound to six ligands, including three conserved histidines and three water molecules, displaying the coordination geometry of a distorted octahedron. One of the water molecules is proposed to play the role of stabilizing the tetrahedral intermediate during the catalysis of SVMPs. The putative $S_{1}^{\prime}$ specificity pocket of TM-3 is relatively shallow, in contrast to the deep pockets of adamalysin $\mathrm{II}$, atrolysin $\mathrm{C}$ and $\mathrm{H}_{2}$-proteinase, but is similar to those in acutolysin A and TACE. The shallow pocket is a consequence of the presence of the non-conserved disulfide bond Cys159-Cys181 and the residue Gln174 at the bottom of the $S_{1}^{\prime}$ pocket. The results indicate that the active-site structure of TM-3, among the know structures of SVMPs examined thus far, is most similar to that of TACE owing to their close disulfide configurations and the $S_{1}^{\prime}$ specificity pocket.

\section{Introduction}

Zinc-dependent metalloendopeptidases (zincins) are widely distributed in various biological systems. They have been classified into three major families, 'gluzincins', 'metzincins' and 'aspzincins', according to the structural topology around the active site (Hori et al., 2001). In metzincins, the typical features of the structure are a $\beta$-turn with a Met residue (Metturn) near the zinc ion, as well as the metal-binding consensus $\mathrm{HE} x x \mathrm{H} x x \mathrm{G} x x \mathrm{H}$ sequence at the active site. Based on the overall structures or the source of origin, metzincins are also grouped into four subfamilies, including adamalysins (also termed snake-venom metalloproteinases; SVMPs), matrixins (vertebrate collagenases, also denoted as matrix metalloproteinases; MMPs), serralysins (large bacterial zinc-endopeptidases) and astacins, with the common presence of a similar zinc-dependent catalytic domain (Stöcker et al., 1995; Stöcker \& Bode, 1995; Bode et al., 1996). In addition, animal tissues have been reported to produce a large number of
Received 4 February 2002 Accepted 28 March 2002

PDB Reference: TM-3, 1kuf, r1kufsf.
C 2002 International Union of Crystallography Printed in Denmark - all rights reserved 
multi-domain proteins, generally called ADAM (a disintegrinlike and metalloproteinase protein), that contain the same central catalytic domain as SVMPs and MMPs (Fox \& Long, 1998; Maskos et al., 1998). ADAMs are present in a wide range of organisms and mammalian tissues and appear to be transmembrane proteins which play important roles in many physiological processes, such as fertilization, neurogenesis and inflammation (Wolfsberg \& White, 1996; Rooke et al., 1996; Tortorella et al., 1999). The similarity of SVMPs, MMPs and ADAMs, along with the high-degree conservation of their tertiary structures at the active sites, suggested that binding of substrates and inhibitors might follow a similar pathway.

A well known example is the $\mathrm{TNF} \alpha$-converting enzyme (TACE), also called ADAM 17, responsible for the cleavage of membrane-bound TNF $\alpha$ precursor at the Ala76-Val77 bond, thus liberating the soluble $\mathrm{TNF} \alpha$ into the extracellular space (Black et al., 1997; Moss et al., 1997). Recently, the crystal structure of the catalytic proteinase domain of TACE was reported and showed similar topology and organization to the structures of the SVMP family, with almost identical orientations of $\alpha$-helices and the central five-stranded $\beta$-sheet (Maskos et al., 1998). Since TNF $\alpha$ has been implicated in various pathological conditions such as rheumatoid arthritis, cachexia and endotoxic shock (Moreland et al., 1997), inhibitors which block the proteolytic activity of TACE may be useful as therapeutic candidates (Barlaam et al., 1999; Xue et al., 2001).

The catalytic mechanism of carboxypeptidase A (CPA), one of the most intensively studied examples of zinc-dependent metalloproteinases, is generally believed to proceed through the promoted-water pathway in which the carboxylic group of Glu270 acts as a general base to promote the attack of a nucleophilic water on the carbonyl group of the scissile peptide bond. The stabilized tetrahedral intermediate then collapses with a requisite proton donation from the protonated Glu270 (Lipscomb \& Sträter, 1996). Tyr248 in CPA, once thought to be the proton donor for the leaving amino group of the scissile peptide bond (Dugas, 1989), was recently proposed to form a Tyr $\mathrm{O}^{\eta} \cdots \mathrm{H}-\mathrm{N}$ hydrogen bond to the departing amino group (Bukrinsky et al., 1998). However, a site-directed mutagenesis study pointed to the possibility that the phenolic hydroxyl of this Tyr248 is hydrogen bonded to the zinc-bound water molecule, which may cause a further activation of the water molecule (Cho et al., 2001). This proposition is consistent with the kinetic data from serralysins, which suggested that the zinc-coordinated Tyr216 deprotonates a water molecule attacking the zinc-activated substrate (Mock \& Yao, 1997). Furthermore, the astacin and an aspzincin GfMEP (a metalloendopeptidase from the mushroom Grifola frondosa) were also reported to possess a Tyr149 and a Tyr133, respectively, holding the zinc ion at the active site (Hori et al., 2001; Yiallouros et al., 2000). These Tyr residues were believed to act as a proton donor to the oxyanion of the tetrahedral intermediate, therefore stabilizing the reaction transition state during catalysis.

In SVMPs, this well studied Tyr residue has never been identified in spite of the elucidation of several crystal struc- tures of this proteinase family (Gomis-Rüth et al., 1994; Zhang et al., 1994; Kumasaka et al., 1996; Gong et al., 1998). Instead, this residue in SVMPs appears to be mainly substituted by Pro or Ala residues which possess no lone-pair electrons on their side chains and are oppositely oriented to Tyr149 in astacin. The active site of SVMPs generally shows a zinc ion which was thought to be tetrahedrally coordinated by three His residues and a water molecule. However, the geometry reported for this zinc ion seems somewhat distorted, especially in the cases of adamalysin II and $\mathrm{H}_{2}$-proteinase, in which the three His $\mathrm{N}^{\varepsilon 2}-\mathrm{Zn}$-water angles are markedly larger than those of His $\mathrm{N}^{\varepsilon 2}-\mathrm{Zn}-$ His $\mathrm{N}^{\varepsilon 2}$ (Gomis-Rüth et al., 1994; Kumasaka et al., 1996). In general, a zinc ion could form complexes with coordination numbers 4,5 and 6 and the coordination geometry of the zinc ion could be very flexible owing to low energy differences between different coordinations (Lipscomb \& Sträter, 1996). The structural data of SVMPs lead to a critical question, i.e. whether one or more of the structural binding water molecules located between the zinc-coordinated water and His residues play(s) some role in stabilizing the tetrahedral intermediate during catalysis, like the Tyr149 in astacin.

In this report, we describe the high-resolution crystal structure of TM-3, an SVMP from Trimeresurus mucrosquamatus venom (Huang et al., 1993, 1995), in which the activesite zinc ion was replaced by a cadmium ion during the crystallization process. The coordination geometry of this cadmium ion and its coordinated ligands are discussed and compared with the currently available structures of other SVMPs. Moreover, we have made a detailed comparison of the active-site structure of TM-3 with those of other SVMPs as well as TACE, human neutrophil collagenase (HNC) and astacin, with particular reference to the $S_{1}^{\prime}$ specificity pocket. Our results indicate that the active-site structure of TM-3 is most similar to that of TACE among the SVMP structures known thus far.

\section{Materials and methods}

\subsection{Crystallization}

TM-3 was isolated from the venom of Taiwan habu (Trimeresurus mucrosquamatus) and purified to high homogeneity as described previously (Huang et al., 1993). Crystals were obtained using crystallization screening kits fromHampton Research (Laguna Niguel, CA, USA). The purified TM-3 (10.5 mg ml ${ }^{-1}$ in $0.2 \mathrm{M}$ ammonium acetate buffer $\mathrm{pH}$ 6.0) was mixed with an equal volume of mother liquor [0.1 $\mathrm{M}$ $\mathrm{CdCl}_{2}, 0.1 M$ sodium acetate and 30\%(v/v) PEG $\left.400 \mathrm{pH} 4.6\right]$ and then crystallized at $277 \mathrm{~K}$ using the hanging-drop vapourdiffusion method. Rod-like crystals started to appear and their dimensions reached $0.4 \times 0.4 \times 3.5 \mathrm{~mm}$ within two weeks. Replacement of $\mathrm{CdCl}_{2}$ with either $\mathrm{ZnCl}_{2}$ or $\mathrm{ZnSO}_{4}$ at the same concentration $(0.1 M)$ in the mother liquor for alternative crystallization experiments was also accomplished in order to obtain crystals without bound cadmium ions. 


\subsection{Data collection, processing and structure refinement}

The 40-1.75 ̊ data set from crystal 1 was collected using a Rigaku R-AXIS IV system at Academia Sinica, Taipei, Taiwan. Diffraction data were processed and scaled using the programs $D E N Z O$ and $S C A L E P A C K$, respectively (Otwinowski \& Minor, 1997) (see Table 1). The 99-1.35 A data set from crystal 2 was obtained using beamline 38B1 at SPring-8, Hyogo, Japan. Data were processed and integrated with the program HKL2000 (Otwinowski \& Minor, 1997). All data collections took place at $123 \mathrm{~K}$. The crystal structure of TM-3 was solved by the molecular-replacement method using the program AMoRe (Navaza, 1994) and a metalloproteinase model, $\mathrm{H}_{2}$-proteinase (atomic coordinates kindly provided by Dr T. Kumasaka). Manual rebuilding and computational refinement were performed using the programs $O$ (Jones et al., 1991) and CNS (Brünger et al., 1998) running on an SGI Octane or $\mathrm{O} 2$ workstations. The parameters for ideal protein geometry of Engh and Huber were used for the refinements (Engh \& Huber, 1991) and the stereochemical quality of the refined structures was checked with the program PROCHECK (Laskowski et al., 1993). In addition, well ordered water molecules were located and included in the model. Both $R$ and $R_{\text {free }}$ were used to monitor the progress of structural refinement.

\subsection{Estimation of the contents of zinc and cadmium ion in TM-3}

The detection of zinc or cadmium ions of TM-3 under different conditions was accomplished by employing an atomic absorption spectrophotometer (Hitachi model Z-8200, Tokyo, Japan) and using A-type graphite cuvettes (Hitachi). For the crystalline state condition, crystals of non-bound TM-3 were pipetted out from the mother liquor and washed with

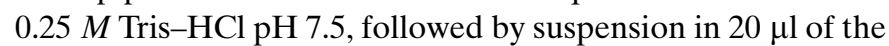
same Tris buffer as a crystal-suspended stock solution. The proteinase concentration was determined by mixing an aliquot of the stock solution with $6.3 \mathrm{M}$ guanidine hydrochloride at pH 7.5 and was spectroscopically analyzed using an extinction coefficient of $26390 \mathrm{M}^{-1} \mathrm{~cm}^{-1}$ at $280 \mathrm{~nm}$ estimated from the amino-acid sequence of TM-3 (Gill \& von Hippel, 1989). When subjected to atomic analysis, aliquots of the crystalsuspended solution were diluted with $0.05 M \mathrm{HNO}_{3}$ to the concentration range within the standard curve. For the native condition, TM-3 dissolved in $0.025 \mathrm{M}$ ammonium acetate $\mathrm{pH}$ 6.0 was directly diluted to appropriate concentrations with $0.05 \mathrm{MHNO}_{3}$. A series of 1.5-7.5 $\mu \mathrm{M} \mathrm{ZnSO}_{4}$ and 2.8-14.2 $\mathrm{n} M$ $\mathrm{CdCl}_{2}$ were used as the standard solutions. The wavelengths for zinc and cadmium absorption were set to 307.6 and $228.8 \mathrm{~nm}$, respectively.

\subsection{Activity assay of $\mathrm{TM}-3$ in the crystalline state}

In order to investigate the proteolytic activity of TM-3 in the crystalline state, the crystal-suspended solution mentioned above was again prepared and the crystals were thoroughly washed with Tris buffer. The activity assay was essentially identical to that of the previous report (Huang et al., 1999)
Table 1

Data-collection and refinement statistics.

Values in parentheses are for the outermost resolution shell.

\begin{tabular}{|c|c|}
\hline \multicolumn{2}{|l|}{ Crystal data } \\
\hline \multicolumn{2}{|l|}{ Unit-cell parameters $(\AA)$} \\
\hline$a=b$ & 60.991 \\
\hline$c$ & 128.398 \\
\hline Space group & $P 4_{1} 2_{2} 2$ \\
\hline Resolution range $(\AA)$ & $\begin{array}{l}99-1.35 \\
\quad(1.40-1.35)\end{array}$ \\
\hline No. of observations & 113041 \\
\hline Unique reflections & 46914 \\
\hline Completeness (\%) & $86.7(82.2)$ \\
\hline Average $I / \sigma(I)$ & $16.9(2.1)$ \\
\hline$R_{\text {merge }} \dagger(\%)$ & $5.7(47.2)$ \\
\hline \multicolumn{2}{|l|}{ Refinement data } \\
\hline No. of reflections $[>0 \sigma(F)]$ & 43,969 \\
\hline$R_{\text {working }} \ddagger$ & 0.181 \\
\hline$R_{\text {free }}(5 \%$ data $)$ & 0.204 \\
\hline R.m.s.d. bond distance $(\AA)$ & 0.012 \\
\hline R.m.s.d. bond angle $\left({ }^{\circ}\right)$ & 1.54 \\
\hline \multicolumn{2}{|l|}{ Average $B$ value/No. of atoms } \\
\hline All non-H atoms & $15.5 / 2022$ \\
\hline Protein & $12.2 / 1616$ \\
\hline Heavy atom & 21.6/10 \\
\hline Water & $28.6 / 396$ \\
\hline \multicolumn{2}{|c|}{ Ramachandran plot (excluding prolines and glycines) } \\
\hline Residues in most favored regions & $167(88.8 \%)$ \\
\hline Residues in additional allowed regions & $20(10.6 \%)$ \\
\hline Residues in generously allowed regions & $1(0.5 \%)$ \\
\hline
\end{tabular}

using FITC casein as the substrate and purified TM-3 $\left(1.4 \mathrm{mg} \mathrm{ml}^{-1}\right)$ as a positive control. The catalytic assays were carried out twice at $310 \mathrm{~K}$ with the addition of different amounts of TM-3 crystals.

\section{Results and discussion}

\subsection{Crystallographically deduced sequence and overall structure of TM-3}

We collected two sets of diffraction data, 40-1.75 and 99$1.35 \AA$, from the tetragonal TM-3 crystals using an image plate and a charge-coupled device (CCD) as detectors, respectively. The crystal structure of TM-3 was solved and preliminarily refined to $1.75 \AA$ resolution using data from the first set, followed by thorough refinement using the second set to $1.35 \AA$ resolution. The final $R$ and $R_{\text {free }}$ values were 0.181 and 0.204 , respectively (Table 1 ).

The primary structure of TM-3 shown in Fig. 1, based on protein sequencing by Edman degradation and cDNA sequence analysis (Huang et al., 1995), was also confirmed by high-resolution crystallography. We have also listed the segments of protein sequence corresponding to the various secondary-structural elements displayed in Fig. 2. Sequence alignment of TM-3 with some other SVMPs reveals that TM-3 is about 47.0, 49.0, 54.0 and $68.3 \%$ identical to acutolysin A, atrolysin $\mathrm{C}$, adamalysin II and $\mathrm{H}_{2}$-proteinase, respectively. 61 of the 200-203 residues are highly conserved among these five sequences, which include those corresponding to the zincbinding consensus segment (residues 143-153) and the Met- 

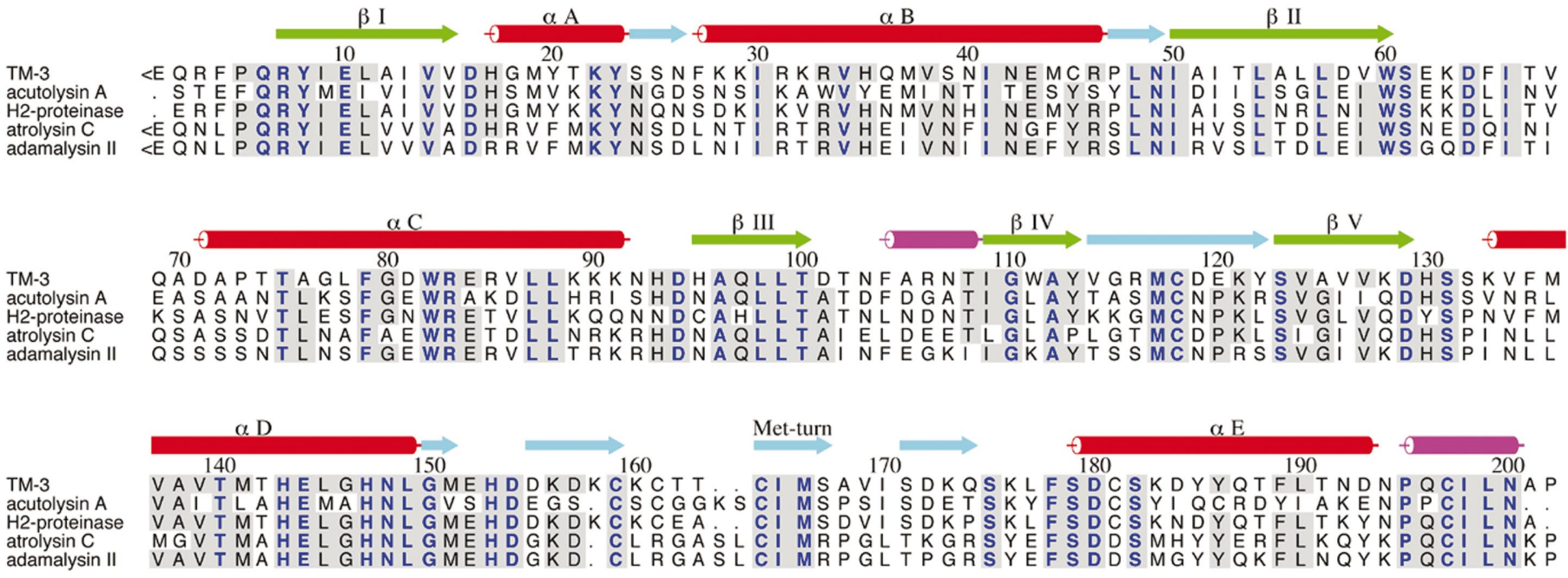

\section{Figure 1}

Alignment of the amino-acid sequence of TM-3 with some snake-venom metalloproteinases. All selected sequences have been confirmed by X-ray crystallography. $<$ E denotes a pyroglutamate group, i.e. 5-pyrrolidone-2-carboxylic acid. The six N-terminal residues and the internal Pro47-Ser61 segment of TM-3 were determined by deblocking and $\mathrm{CNBr}$ digestion followed by the Edman degradation method, while the others were deduced from the cDNA clone of mpts-8 (Huang et al., 1995), which is checked and confirmed mostly by crystallography. Secondary-structure elements were defined by PROCHECK. The green and cyan arrows indicate the locations of $\beta$-strands and turns, respectively, in the crystal structure of TM-3. The red and purple cylinders represent $\alpha$-helices and $3_{10}$-helices, respectively. Residue numbering refers to that of TM-3. Residues that have over $80 \%$ identity in these five sequences are shaded and the 61 highly conserved residues are labelled in blue with bold characters. The figure was prepared using ALSCRIPT. Acutolysin A is a haemorrhagic metalloproteinase isolated from the venom of Chinese mainland Agkistrodon acutus (Gong et al., 1998). $\mathrm{H}_{2}$-proteinase is a non-haemorrhagic proteinase from T. flavoviridis venom (Takeya et al., 1989). Atrolysin C is a weaker haemorrhagic toxin from the the Western diamondback rattlesnake Crotalus atrox (Shannon et al., 1989), while adamalysin II is the non-haemorrhagic proteinase from the Eastern diamondback rattlesnake C. adamanteus (Gomis-Rüth et al., 1994).
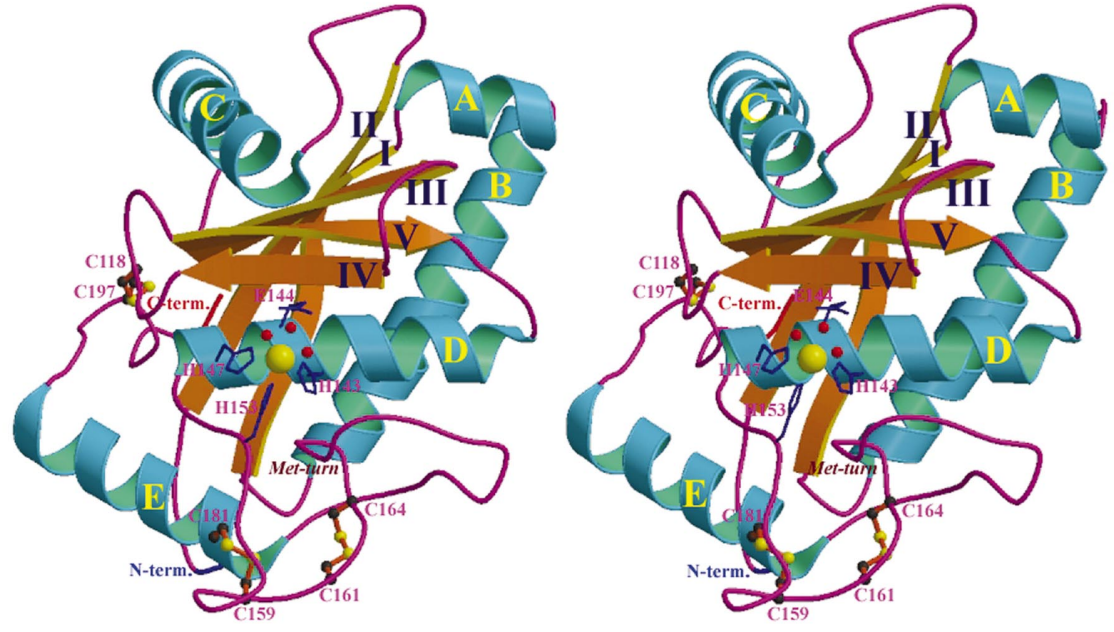

Figure 2

A ribbon diagram of the overall structure of TM-3. The stereoview faces towards the active-site cleft. Cadmium ion and its coordinated water molecules in the active site are depicted as yellow and red spheres, respectively. The coordinated histidines and catalytic glutamyl residue are denoted in blue by a stick model. The three disulfide bridges are drawn in orange as a ball-andstick model. In addition, the locations of $\alpha$-helices (A-E), $\beta$-strands (I-V) and the methionineturn as well as the $\mathrm{N}$ - and $\mathrm{C}$-terminal residues are also indicated. The figure was produced using MOLSCRIPT.

turn (residues 164-166). We noted that the amino-acid sequence of TM-3 identified by X-ray crystal structure (Fig. 1) appears to show three residue differences, i.e. Tyr20, Ile41 and Ala168, compared with that derived from the open-reading frame of the cDNA clone reported previously (Huang et al., 1995). This variation could be a consequence of the poly- morphism arising from different sample sources or the existence of variant forms of this metalloproteinase.

The overall structure of TM-3, as depicted in Fig. 2, exhibits the typical structural features of SVMPs; namely, an oblate ellipsoidal molecule composed of a central five-stranded $\beta$-sheet mixed with five $\alpha$-helices in a unique arrangement $\beta \mathrm{I}$ $\alpha \mathrm{A}-\alpha \mathrm{B}-\beta \mathrm{II}-\alpha \mathrm{C}-\beta \mathrm{III}-\beta \mathrm{IV}-\beta \mathrm{V}-\alpha \mathrm{D}-\alpha \mathrm{E}$ and a highly conserved methionine-containing turn (Met-turn), i.e. the segment Cys164Ile165-Met166, lying between $\alpha \mathrm{D}$ and $\alpha \mathrm{E}$. The protein structure consists of two domains separated by a cleft. The upper domain (about 150 N-terminal residues) has an open-sandwich topology comprising the twisted five-stranded $\beta$-sheet with $\alpha$-helices distributed on both sides, i.e. $\alpha \mathrm{A}, \alpha \mathrm{B}$ and $\alpha \mathrm{D}$ being on one side and $\alpha \mathrm{C}$ on the other. The lower domain (about $50 \mathrm{C}$-terminal residues) is composed of $\alpha \mathrm{E}$ plus some coils and turns, including the above-mentioned Met-turn.

Strand IV of the central $\beta$-sheets, antiparallel to the other strands, faces the active-site cleft where the zinc-binding site is located. The environment of this metal-binding site is topologically very similar to those of other reported metzincins (Figs. $3 a$ and $3 b$ ), all of which include a zinc-binding consensus 
segment, $\mathrm{H}^{143} \mathrm{E}^{144} x x \mathrm{H}^{147} x x \mathrm{G}^{150} x x \mathrm{H}^{153}$, located near the end of the $\alpha \mathrm{D}$ helix. In contrast to other SVMPs with two disulfide cross-linkages, such as adamalysin II and atrolysin C isolated from the venoms of American diamondback rattlesnakes (Gomis-Rüth et al., 1994; Zhang et al., 1994), the TM-3 structure reported here possesses three disulfide linkages, Cys118-Cys197, Cys159-Cys181 and Cys161-Cys164, with the latter apparently being used for stabilizing the loose Met-turn conformation. The extra disulfide Cys159-Cys181 was also identified in the structure of $\mathrm{H}_{2}$-proteinase and acutolysin $\mathrm{A}$, two small SVMPs isolated from the venoms of Japanese habu (T. flavoviridis) and Chinese mainland Agkistrodon acutus, respectively (Kumasaka et al., 1996; Gong et al., 1998).

\subsection{The geometry of the environment surrounding the active-site metal ion}

The zinc-binding environment, located at the bottom of the relatively flat active-site cleft, appears to contain a heavy atom $\left(\mathrm{Cd}^{2+}\right)$ ligated by the three imidazole $\mathrm{N}^{\varepsilon 2}$ atoms of the histidine residues His143, His147 and His153 (Fig. 4). Additionally, in contrast to the single $\mathrm{Zn}$-coordinated water molecule identified in the active site of most zinc-dependent metalloproteinases, e.g. Wat300 in astacin, adamalysin II and acutolysin A (Fig. 4b), three water molecules, Wat359, Wat416 and Wat418, were found to coordinate to the $\mathrm{Cd}^{2+}$. Wat359, bridging the $\mathrm{Cd}^{2+}$ and the neighbouring Glu144, is tightly bound as judged by its low temperature factor $\left(13.3 \AA^{2}\right.$ compared with the global average of $28.6 \AA^{2}$ ). Presumably, Wat359 is equivalent to the reported Wat300 which is believed to be the nucleophilic water molecule proposed in the catalysis mechanism of carboxypeptidase A. The contact distances Glu144 $\mathrm{O}^{\varepsilon 1}$-Wat359 O and Glu144 $\mathrm{O}^{\varepsilon 2}$-Wat359 $\mathrm{O}$ are shorter (2.94 $\AA$ and $3.08 \AA$, respectively) than those of other SVMPs (Gomis-Rüth et al., 1994; Zhang et al., 1994; Kumasaka et al., 1996; Gong et al., 1998).

During the structural refinements, the active-site $\mathrm{Cd}^{2+}$ of TM-3 possesses a temperature factor of $9.2 \AA^{2}$, comparable to those of its coordinated imidazole $\mathrm{N}$ atoms, which are $6.8,7.8$ and $8.6 \AA^{2}$, respectively. However, it seems too low if $\mathrm{Zn}^{2+}$ $\left(4.3 \AA^{2}\right)$ is used as the active-site metal ion. Furthermore, the distances between the zinc ion and the $\mathrm{N}^{\varepsilon 2}$ atom of its ligated histidines in other structures of SVMP are usually shorter than $2.10 \AA$ ( $2.07 \AA$ on average). In the crystal structure of TM-3, the corresponding distances between the metal ion and its ligated histidines are longer than $2.24 \AA$ ( $2.26 \AA$ on average), which lies within the normal range of distance between a cadmium ion and its coordinated $\mathrm{N}$ atoms.

In addition, the presence of $\mathrm{Cd}^{2+}$ in the crystal structure was also confirmed by atomic absorption analysis. As indicated in Table 3, one molecule of native TM-3 contains approximately one $\mathrm{Zn}$ atom, but no $\mathrm{Zn}$ atom was detected in the crystal of this report. This spectrophotometric and crystallographic evidence corroborated the fact that the active-site zinc ion of TM-3 had been replaced by a cadmium ion during crystallization. The slightly higher $B$ value for this cadmium compared with those of its surrounding ligand atoms may be a

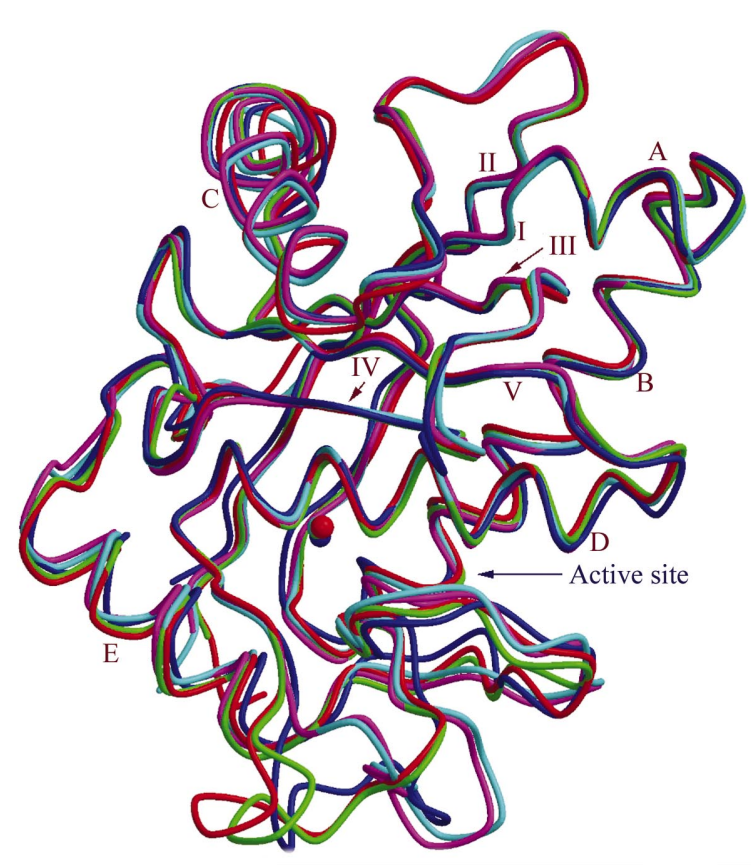

(a)

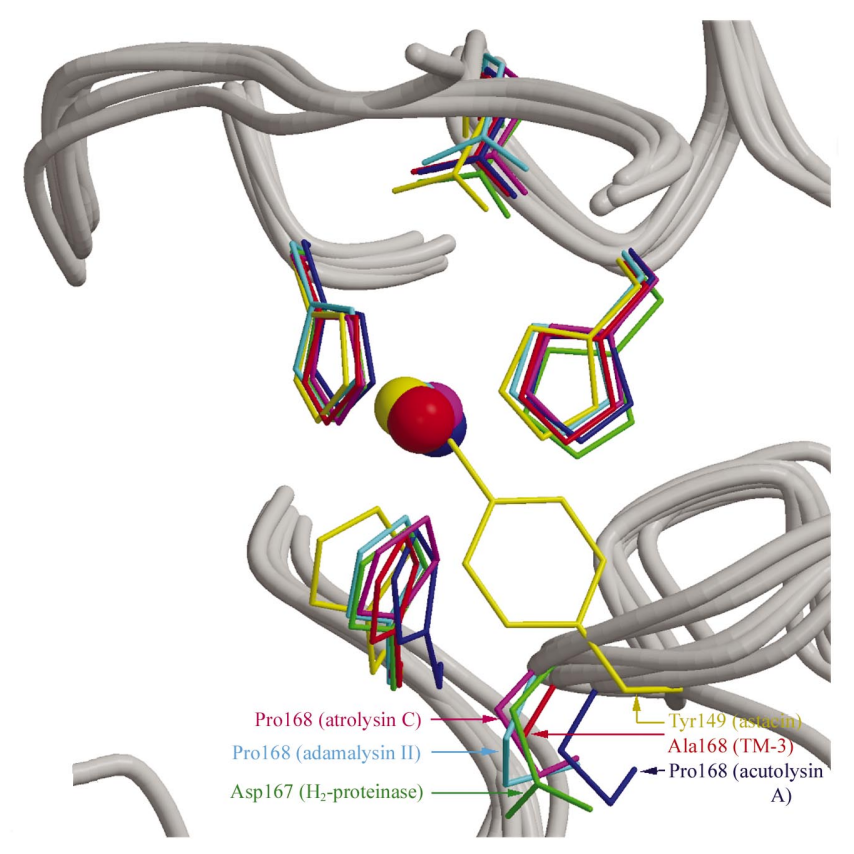

(b)

Figure 3

Superimposition of the TM-3 structure with some snake-venom metalloproteinases. (a) The overall backbone structures are shown. Molecules are as follows: red, TM-3; green, $\mathrm{H}_{2}$-proteinase (Kumasaka et al., 1996); blue, acutolysin A (Gong et al., 1998); magenta, atrolysin C (Zhang et al., 1994); cyan, adamalysin II (Gomis-Rüth et al., 1994). The locations of $\alpha$-helices (A-E) and $\beta$-strands (I-V) indicated refer to Figs. 1 and 2. (b) The active-site clefts are shown. Crayfish astacin (yellow) is also included in addition to those proteinase structures (Bode et al., 1992). The active-site residues and metal ion of each molecule are drawn as a stick model and a ball, respectively, using the same color for each proteinase. The residues in each sequence corresponding to the Tyr149 of astacin or equivalent residues in other proteinases are also indicated. All figures were produced using MOLSCRIPT. 
consequence of the fact that in our crystals there is still a limited content of $\mathrm{Zn}^{2+}$ occupying this site.

The coordination geometry of this cadmium ion displays a distorted octahedron, with His143 $\mathrm{N}^{\varepsilon 2}$, His153 $\mathrm{N}^{\varepsilon 2}$, Wat359 and Wat416 forming an octahedral base plane, and His147 $\mathrm{N}^{\varepsilon 2}$ and Wat418 being located at the vertices (see Table 2 and Fig. 4). Notice that the positions of the three histidine ligands show no significant changes compared with those of reported structures of SVMP in which the zinc ion was claimed to display a distorted tetrahedral geometry (Fig. 3b). The
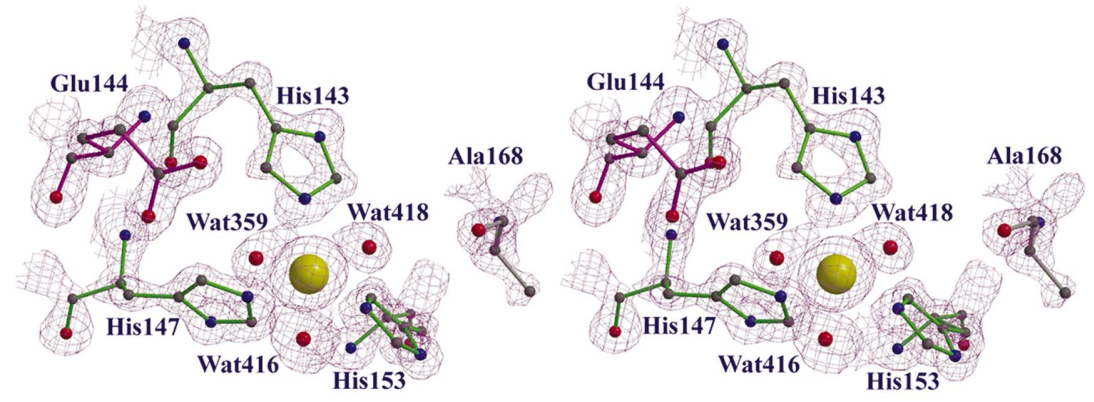

(a)

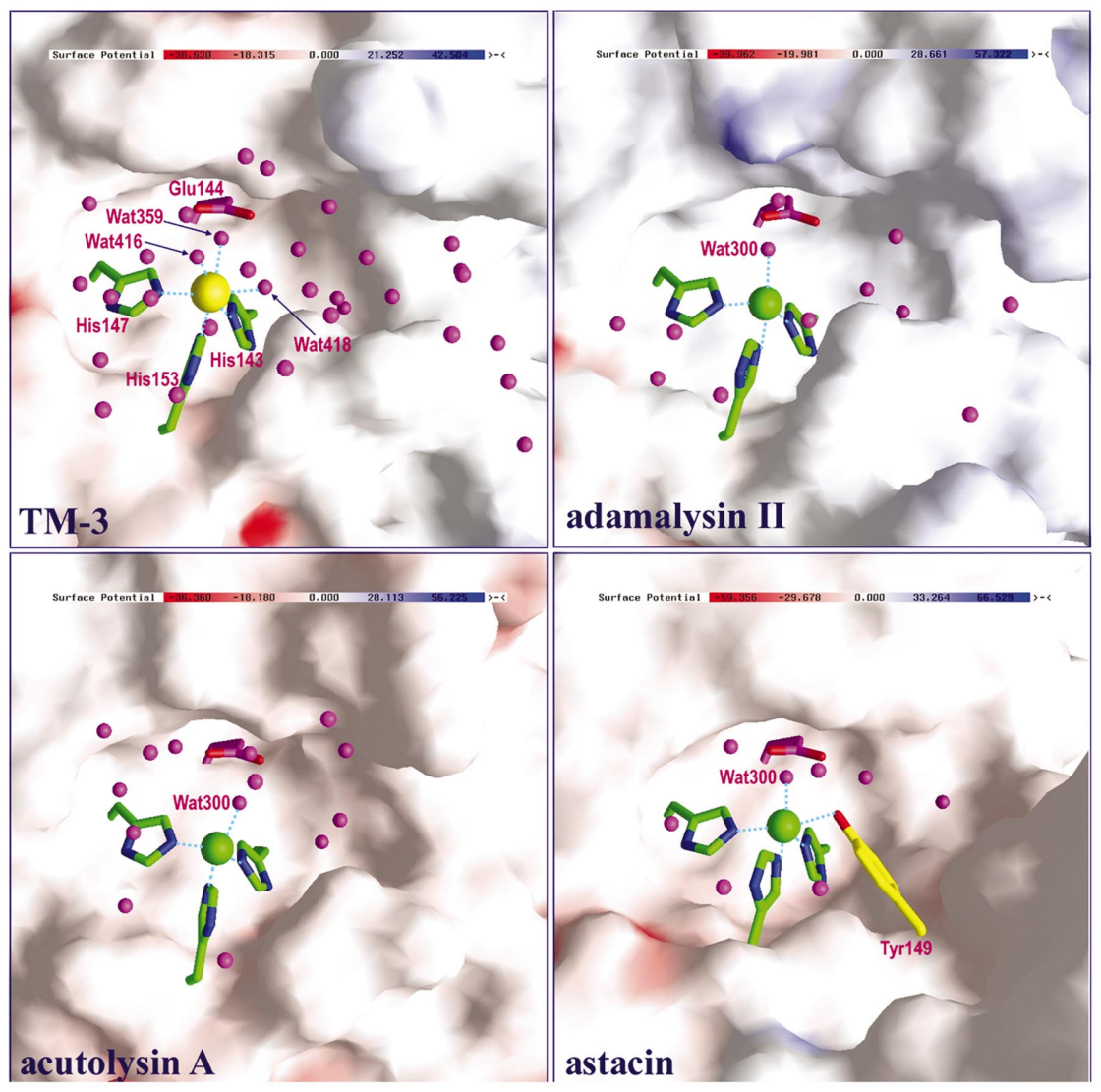

(b)

\section{Figure 4}

The active-site structure of TM-3. (a) A stereoview of the $2 F_{o}-F_{c}$ map (contoured at the $2.35 \sigma$ level) of TM-3 around the active-site structure. Positions of various residues in the active site are indicated and referred to those in Fig. 2. (b) Comparisons of the active-site structures of TM-3, adamalysin II, acutolysin A and astacin. Zinc ions and water molecules are shown as various spheres in green and magenta, respectively. The Tyr149 in astacin is indicated as a stick model in yellow. The figures were prepared using BOBSCRIPT (a) and GRASP $(b)$. proteolytic activity of Cd-bound TM-3 in the crystalline state towards the casein substrate still persisted despite a lower activity (about $5-10 \%$ ) compared with the native TM-3 in solution (data not shown). As shown in Fig. 4(b), our highresolution (1.35 $\AA$ ) structure of TM-3 is likely to lead to the identification of more water molecules in the active site compared with the structures of adamalysin II (2.0 $\AA$ resolution) and acutolysin A (1.9 $\AA$ resolution). Of these water molecules, we are particularly interested in the role of the two additional Cd-bound waters, Wat416 and Wat418. Whether the presence of these two water molecules arises from the larger van der Waals dimension of the cadmium ion which keeps the mobilities of its ligands low or from the high-resolution diffraction of our crystals remains to be answered. Reminiscently, there were two Zn-coordinated water molecules detected in the active site of a monoclinic aspzincin structure which was refined to $1.6 \AA$ resolution (Hori et al., 2001).

Wat418 of TM-3 occupies a position approximately corresponding to that of the hydroxyl group of Tyr149 in astacin (Fig. 4b). In general, the $\mathrm{Zn}$-coordinated Tyr residue in the structures of astacin and the aspzincin $G f$ MEP have been believed to act as a proton donor to the oxyanion of the tetrahedral intemediate in the catalytic processes (Hori et al., 2001; Yiallouros et al., 2000). However, so far no such Tyr residue has been identified in the active site of SVMP structures. According to a report by Lipscomb (1974), the $\mathrm{p} K_{\mathrm{a}}$ value of $\mathrm{Zn}$-bound water in carboxypeptidase is approximately 8.9 , lower than that of the commonly observed Tyr side chain $\left(\mathrm{p} K_{\mathrm{a}}=10.13\right)$. Thus, water molecules could functionally display the role of Tyr residue in the formation of a hydrogen bond. We think that the reaction transition state during catalysis of SVMPs is possibly stabilized by a second Zn-bound water molecule which is located between the first catalytic water molecule and one of the three Zn-coordinated histidines, similar to the hydroxyl group of Tyr149 in astacin.

\subsection{Configuration of disulfide bridges and the $S_{1}^{\prime}$ pocket}

As shown in Figs. 1 and 2, the segment Gly150-Ser179, connecting $\alpha \mathrm{D}$ with $\alpha \mathrm{E}$, consists of complicated turns and loops stabilized by the disulfide linkages Cys159Cys181 and Cys161-Cys164. About half of this segment surrounds the active-site region and thus dictates the conformation of substrate-binding pocket, especially in the 
Table 2

Coordination geometry of the active-site cadmium ion.

\begin{tabular}{lllr}
\hline Bond lengths $(\AA)$ & & Bond angles $\left(^{\circ}\right)$ & \\
\hline His143-Cd & 2.27 & His143-Cd-His147 & 90.76 \\
His147-Cd & 2.28 & His143-Cd-His153 & 103.53 \\
His153-Cd & 2.24 & His147-Cd-His153 & 96.51 \\
Wat359-Cd & 2.30 & His143-Cd-Wat359 & 93.68 \\
Wat416-Cd & 2.31 & His143-Cd-Wat416 & 158.13 \\
Wat418-Cd & 2.24 & His143-Cd-Wat418 & 87.11 \\
& & His147-Cd-Wat359 & 94.49 \\
& & His147-Cd-Wat416 & 95.77 \\
& & His147-Cd-Wat418 & 171.15 \\
& & His153-Cd-Wat359 & 159.39 \\
& & His153-Cd-Wat416 & 96.45 \\
& & His153-Cd-Wat418 & 92.34 \\
& & Wat359-Cd-Wat416 & 65.07 \\
& & Wat359-Cd-Wat418 & 77.09 \\
& & Wat416-Cd-Wat418 & 83.24 \\
\hline
\end{tabular}

$\mathrm{S}_{1}^{\prime}$ specificity subsite (Fig. 5). Interestingly, this segment appears to be more diverse and variable between TM-3 and the other four reported structures of SVMP, except for the conserved Met-turn (Figs. 1 and 3), even though they share a similar overall structure. For acutolysin A, which is fully superimposable in its disulfide configurations with TM-3, the relative size and dimension of the $S_{1}^{\prime}$ pocket is very similar, except that a slight extension in the latter structure is found (Figs. 5a and 6). In adamalysin II and atrolysin C, only one disulfide bridge, Cys157-Cys164, was identified in the region corresponding to Gly150-Ser179 of TM-3 (Gomis-Rüth et al., 1994; Zhang et al., 1994). In contrast to TM-3, residues 172176 in both the adamalysin II and atrolysin C structures appear to move away from $\alpha \mathrm{E}$ (see Figs. $3 a$ and $5 b$ ). These conformational changes make the $S_{1}^{\prime}$ pocket of these two proteinases significantly deeper than that of TM-3 (Fig. 6). However, as shown in Fig. 6, $\mathrm{H}_{2}$-proteinase appears to have a more extended $S_{1}^{\prime}$ pocket compared with that of TM-3, although all disulfide linkages are topologically identical to those of our structure. This is attributed to the substitution of Gln174 in TM-3 by Pro173 in $\mathrm{H}_{2}$-proteinase, removing the relatively bulky side chain of Gln (Fig. 5a). Also, Gln174 of TM-3 is shown to correspond to Thr174 in acutolysin A; both possess a similar orientation of their side chains (Fig. 5a).

Adamalysin II and $\mathrm{H}_{2}$-proteinase are both non-haemorrhagic and atrolysin $\mathrm{C}$ has a weak haemorrhagic activity (Takeya \& Iwanaga, 1998). In contrast, acutolysin A has a strong haemorrhagic activity. Based on structural and functional comparisons, the haemorrhagic activity of a particular SVMP seems to be related to the dimension/depth of its $S_{1}^{\prime}$ specificity pocket. However, according to a phylogenetic study (Tsai et al., 2000), TM-3 should be weakly haemorrhagic or non-heamorrhagic and is likely to depart from the presumed correlation between haemorrhagic activity and the $S_{1}^{\prime}$ pocket. In addition, based on sequence alignments, a number of SVMPs with strong haemorrhagic activity have a Pro residue equivalent to Pro173 in $\mathrm{H}_{2}$-proteinase (Takeya \& Iwanaga, 1998; Tsai et al., 2000). Thus, the $S_{1}^{\prime}$ pockets of these SVMPs should be as deep as that of the non-haemorrhagic $\mathrm{H}_{2}$ proteinase. This is also contradictory to our proposed
Table 3

Zinc and cadmium ion contents of TM-3 in the native and crystalline conditions.

\begin{tabular}{lll}
\hline Condition & Zinc ion & Cadmium ion \\
\hline Native & $1.164 \pm 0.018 \dagger$ & $0.000 \pm 0.000 \ddagger$ \\
Crystalline & $0.028 \pm 0.047$ & $4.376 \pm 0.052$ \\
\hline
\end{tabular}

$\dagger$ The data, termed as ions per protein molecule, are the average from two independent experiments with different proteinase concentration in the range of the standard curve. Each analysis was carried out at least twice. \$ The absorbance at the cadmium-ion wavelength $(228.8 \mathrm{~nm})$ was lower than that of a blank solution $\left(0.05 M \mathrm{HNO}_{3}\right)$.

haemorrhagicity- $S_{1}^{\prime}$ rule. Hence, in order to delineate the structure-function relationship, an accumulation of solved structures of various SVMPs is warranted.

Alternatively, TM-3 was shown to have a low hydrolytic activity $\left(k_{\text {cat }} / K_{m}=1.70 \times 10^{-4} \mu M^{-1} \mathrm{~s}^{-1}\right)$ toward the casein substrate compared with its variant forms TM-1 and TM-2 $\left(k_{\text {cat }} / K_{m}\right.$ are $7.03 \times 10^{-4}$ and $7.32 \times 10^{-4} \mu M^{-1} \mathrm{~s}^{-1}$, respectively) isolated from the same venom (unpublished data from our group). This raises an interesting question. Is the proteolytic activity of a SVMP related to the dimension and shape of its $S_{1}^{\prime}$ pocket? This question can only be answered by many comparative studies of the structure-activity relationships of SVMPs, including TM-1 and TM-2.

\subsection{Structural comparison of TM-3 with TACE, human neutrophil collagenase and astacin}

Previously, adamalysin II was considered to be a good starting model in the SVMP family for the rational design of drugs against TACE-associated pathological disorders and, indeed, several inhibitors have been synthesized to bind at the active site of TACE based on the crystal structure of adamalysin II (Cirilli et al., 1997; Gomis-Rüth et al., 1998; D’Alessio et al., 1999). However, the crystal structure of the catalytic proteinase domain of TACE showed that TACE possesses three disulfide bridges (Maskos et al., 1998). Topologically, the Cys423-Cys453 bond of TACE is equivalent to the Cys159Cys181, Cys158-Cys180 and Cys157-Cys181 bonds in TM-3, $\mathrm{H}_{2}$-proteinase and acutolysin $\mathrm{A}$, respectively, but no equivalent disulfide bond was found in adamalysin II or atrolysin C. Not unexpectedly, the $S_{1}^{\prime}$ pocket of the TACE structure, while similar to those of TM-3 and acutolysin A, is significantly more shallow than those of adamalysin II and atrolysin $\mathrm{C}$ owing to the additional Cys423-Cys453 bond (Fig. 6). Moreover, Val440 of TACE is shown to be structurally equivalent to Gln174 in TM-3, in terms of forming a similar barrier at the bottom of the $\mathrm{S}_{1}^{\prime}$ pocket (Fig. 5c). Thus, TM-3 and acutolysin A are perhaps better models for the design of drugs against TACEassociated disorders than adamalysin II.

Also shown in Fig. 6, the $S_{1}^{\prime}$ pocket conformations of astacin and human neutrophil collagenase (HNC, also termed MMP8) appear to be quite different from that of TM-3. This is caused by the relatively different topology and organization between TM-3, astacin and HNC structures in the segment Gly150-Ser179 of TM-3 and its corresponding regions (Stams et al., 1994; Bode et al., 1992). Furthermore, TM-3 exhibits a similar overall active-site structure to TACE and HNC (Fig. 7), 

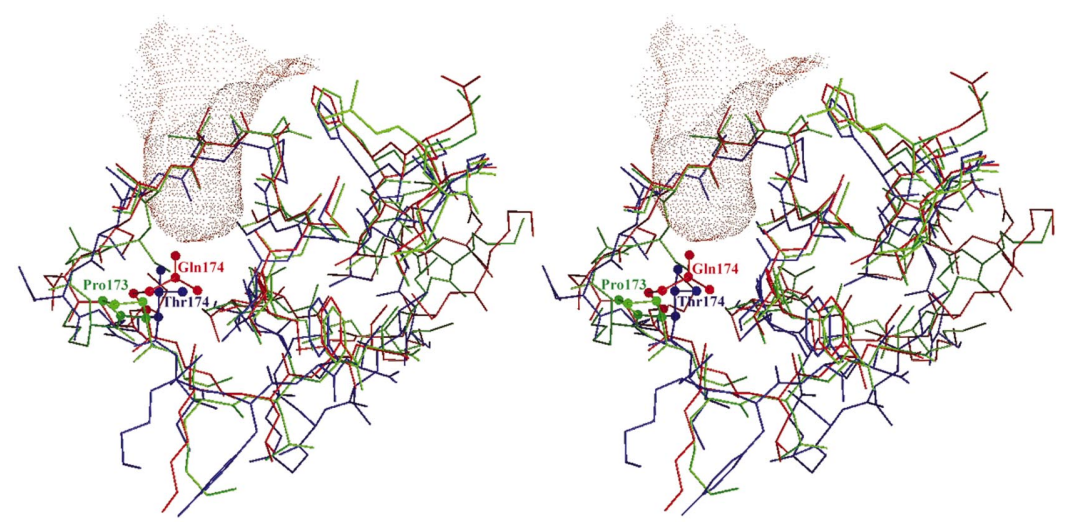

(a)
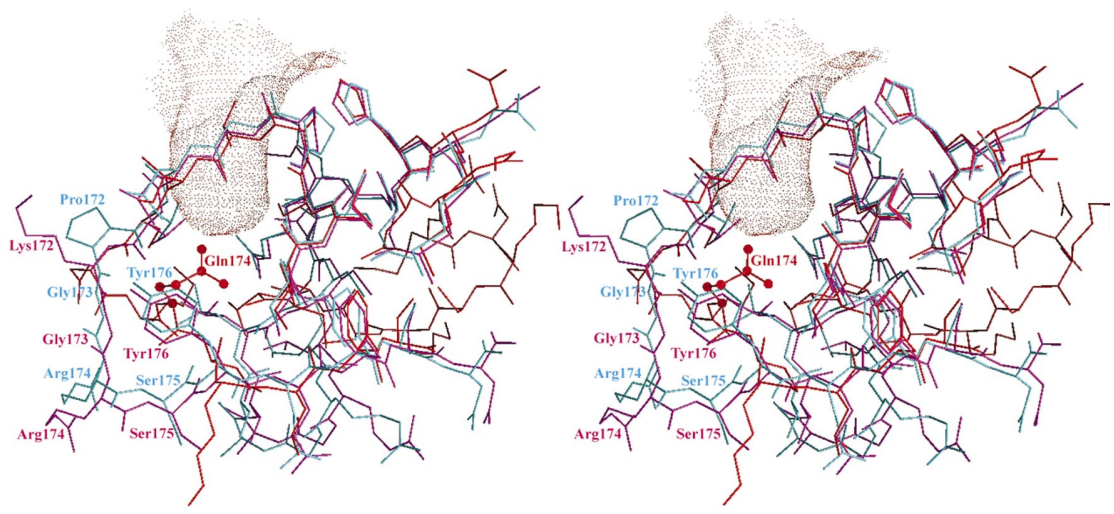

(b)
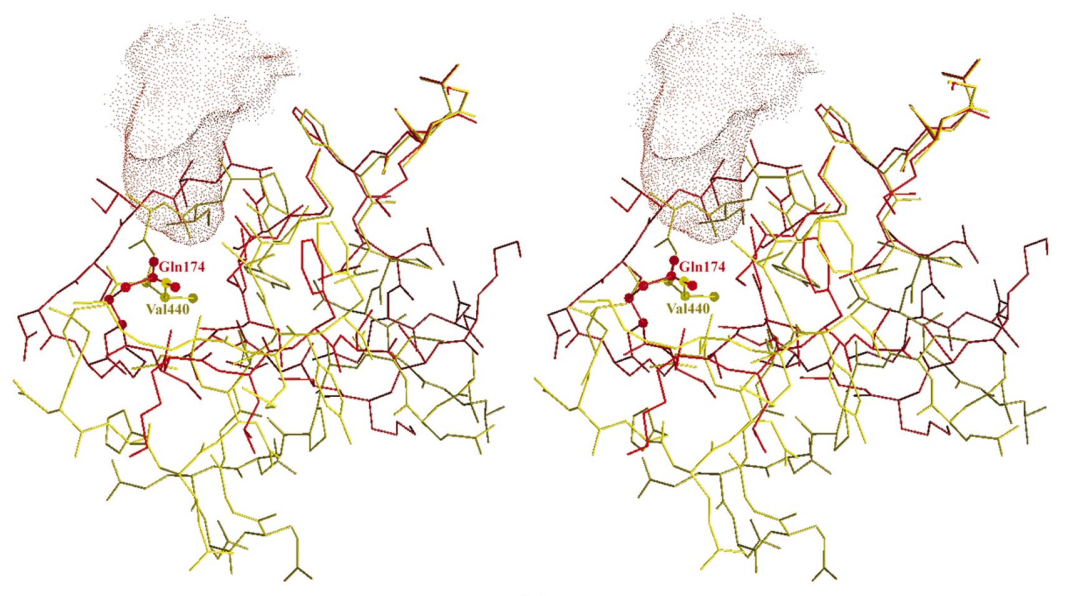

(c)

Figure 5

Structural comparisons of TM-3 with some reported SVMPs and TACE in the region of the segment Gly150-Ser179. The structure of TM-3 is superimposed with those of $(a) \mathrm{H}_{2}$ proteinase and acutolysin A, (b) adamalysin II and atrolysin C or (c) TACE (Maskos et al., 1998). Residues are depicted as stick models, colored red (TM-3), green $\left(\mathrm{H}_{2}\right.$-proteinase), blue (acutolysin A), cyan (adamalysin II), purple (atrolysin C) and yellow (TACE). Gln174 of TM3 and its corresponding residues in $\mathrm{H}_{2}$-proteinase, acutolysin $\mathrm{A}$ and TACE are denoted as ball-and-stick models. The surface of the $\mathrm{S}_{1}^{\prime}$ pocket of TM-3 is also drawn in red dots. Diagrams are stereoviews and were prepared using GRASP.

within which the $S_{1}^{\prime}-S_{3}^{\prime}$ substrate-binding subsites are comparable between these three structures. An additional difference is found at the hydrophobic $\mathrm{S}_{3}^{\prime}$ subsite of TACE, which is slightly deeper than that of TM-3, while that of HNC is relatively shallow and broad (Fig. 7).

\subsection{The other possible cadmium ions}

In addition to the active-site cadmium ion, there are nine other heavy atoms identified in the crystal structure of TM-3 (Table 1 and Fig. 8), which are presumed to be cadmium ions. Five of these heavy atoms, including the active-site atom, are ligated by more than two non-water ligands, such as His, Asp and Glu. They tend to be embedded in the interior of protein molecule and become quite immobilized (average temperature factor $=15.1 \AA^{2}$ ), consistent with the reports of some cadmiumbinding glycoproteins (Meisch \& Schmitt, 1986; Nair \& Robinson, 1999). In general, the imidazole $\mathrm{N}$ atom of histidine, the negative carboxylic group of aspartic and glutamic acid, as well as the amide $\mathrm{N}$ and $\mathrm{O}$ atoms of asparagine and glutamine, are the primary metal-chelation sites for cadmium-binding proteins. Our Cd-containing proteinase crystal is also confirmed by atomic absorption analysis, which indicated that TM-3 crystals of this report contain about four or five cadmium ions per proteinase molecule (Table 3). As for the locations of other heavy atoms, they are mostly found on the surface of the molecule, so that these atoms appear to be less restricted and more flexible (average temperature factor $=31.8 \AA^{2}$ ). Some of these atoms are located near the twofold symmetric centres between two proteinase molecules and usually do not possess full occupancy. These cadmium ions may play some role in facilitating the packing of proteinase molecules during crystallization, as replacement of cadmium chloride with zinc chloride or sulfate failed to produce proteinase crystals.

\section{Conclusions}

We report here the high-resolution crystal structure of TM-3 isolated from the venom of Taiwan habu. The TM-3 structure exhibits the typical structural features of SVMP and possesses three disulfide cross-linkages, including the non-conserved Cys159-Cys181, which is topologically equivalent to Cys158Cys180, Cys157-Cys181 and Cys423-Cys453 in $\mathrm{H}_{2}$-proteinase, acutolysin $\mathrm{A}$ and TACE, respectively. The active-site zinc ion of TM-3 was replaced by a cadmium ion during crystallization. The coordination geometry of this cadmium ion displays a distorted octahedron, with the positions of its three histidine ligands showing no significant changes compared with those of reported SVMP structures in which the zinc ion was thought to have a distorted tetrahedron geometry. 


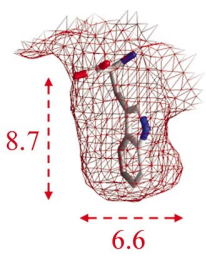

TM-3

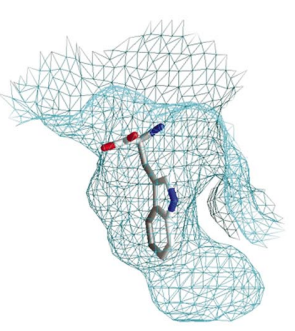

Atrolysin C

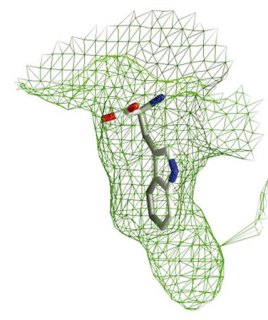

$\mathrm{H}_{2}$-proteinase

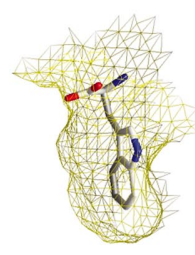

TACE

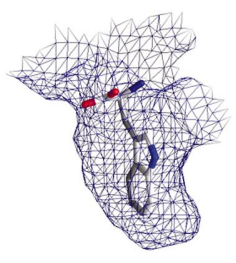

Acutolysin A

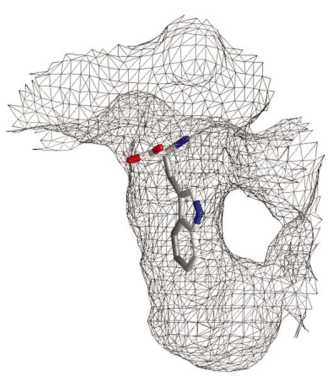

$\mathrm{HNC}$

Figure 6

Comparisons of the $\mathrm{S}_{1}^{\prime}$ pocket of TM-3 with various zinc-dependent metalloproteinases from the SVMP, ADAM, MMP and astacin family. All the pockets are drawn on the same scale. A tryptophan, denoted as a stick model, was modelled into each pocket as a dimension reference. The pocket diameters of TM-3 are indicated in $\AA$. The figure was produced using GRASP. TACE, the catalytic domain of TNF $\alpha$-converting enzyme; HNC, the catalytic domain of human neutrophil collagenase (Stams et al., 1994).

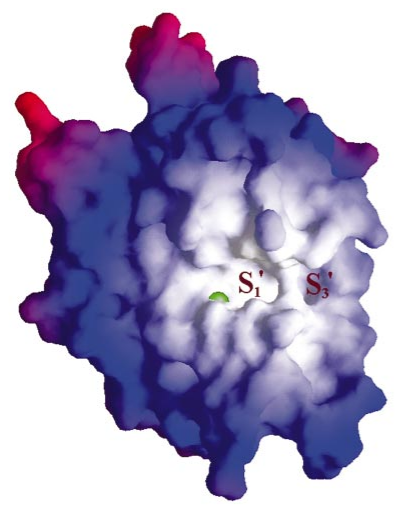

TACE

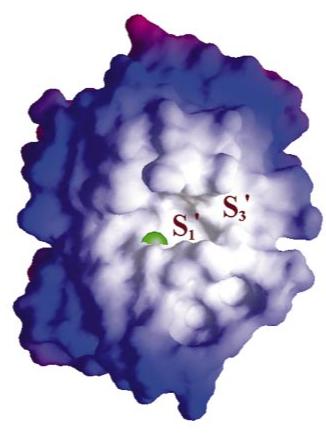

TM-3

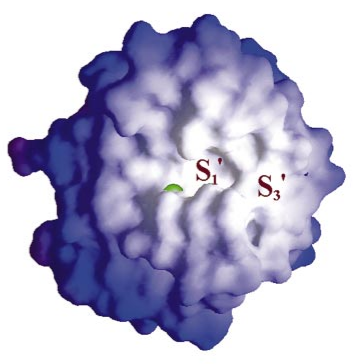

HNC

Figure 7

Solid-surface representation of the structures of TM-3 and the catalytic domains of TNF $\alpha$ converting enzyme (TACE) and human neutrophil collagenase (HNC). The active-site metal ions (green spheres) and the primed substrate-binding subsites are indicated. Distance of various surfaces to the active-site centre are contoured from 0 (light) to $38 \AA$ (dark). These diagrams are shown on the same scale; the figure was produced using GRASP.

The segment Gly150-Ser179 surrounding the active-site region is found to form and dictate the conformation of the substrate-binding pocket, particularly of the $S_{1}^{\prime}$ subsite. The putative $S_{1}^{\prime}$ specificity pocket of TM-3 is relatively shallower than those of $\mathrm{H}_{2}$-proteinase, adamalysin II and atrolysin $\mathrm{C}$ because of the presence of the disulfide bond Cys159-Cys181 in the TM-3 structure and the substitution of Gln174 of TM-3 by Pro173 in $\mathrm{H}_{2}$-proteinase. It is noted that acutolysin $\mathrm{A}$ and

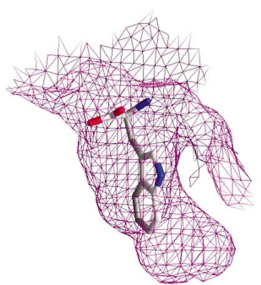

Adamalysin II

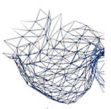

Astacin
TACE contain Thr174 and Val440, respectively, which are structurally equivalent to Gln174 of TM-3 in terms of forming the bottom of the $S_{1}^{\prime}$ subsite. Consequently, the $S_{1}^{\prime}$ specificity pockets among these three proteinases are similar. This report indicates that our structure has a stronger resemblance among the known structures of SVMP to the crystal structure of catalytic domain of $\mathrm{TNF} \alpha$-converting enzyme (TACE), especially in the disulfide configurations and the $S_{1}^{\prime}$ specificity pocket.

So far, native human TACE had been purified from the membranes of the human monocytic cell line THP-1 (Black et al., 1997). Its truncated recombinant forms were also obtained from the expression of TACE cDNAs cloned from the cDNA libraries of human leukocyte, monocyte or epithelial cell (Black et al., 1997; Moss et al., 1997). However, their isolation and purification procedures were usually complicated and the active TACE was only obtained in small amounts. In contrast, SVMPs are abundant in the venoms of crotalid and viperid snakes. Thus, this leads to the possibility of creating an alternative assay system using SVMPs, which can be easily isolated and used for analysis of the TACE inhibitors. Furthermore, many naturally occurring SVMP inhibitors, e.g. endogenous peptidic inhibitors in the venom secretions (Robeva et al., 1991; Francis \& Kaiser, 1993; Huang et al., 1998), and several proteinaceous inhibitors in the sera of the snake itself or some mammals (Catanese \& Kress, 1992; Yamakawa \& Omori-Satoh, 1992; Huang et al., 1999) were shown to specificially and effectively inhibit the proteolytic activities of SVMPs. These inhibitors may be useful as drug candidates for the treatment of TACE-associated inflammatory diseases, owing to the close structural similarity between TACE and SVMPs. The TACE-catalyzed product tumour necrosis factor- $\alpha(\mathrm{TNF} \alpha)$ has been reported to play a beneficial role as an immunostimulant and an important mediator of host resistance to many infectious agents and, probably, malignant tumours (Vilcek \& Lee, 1991). Whether some of the SVMPs or their modified derivatives can functionally substitute the role of TACE in patients with TACE-deficiency diseases deserves to be investigated in the future. 


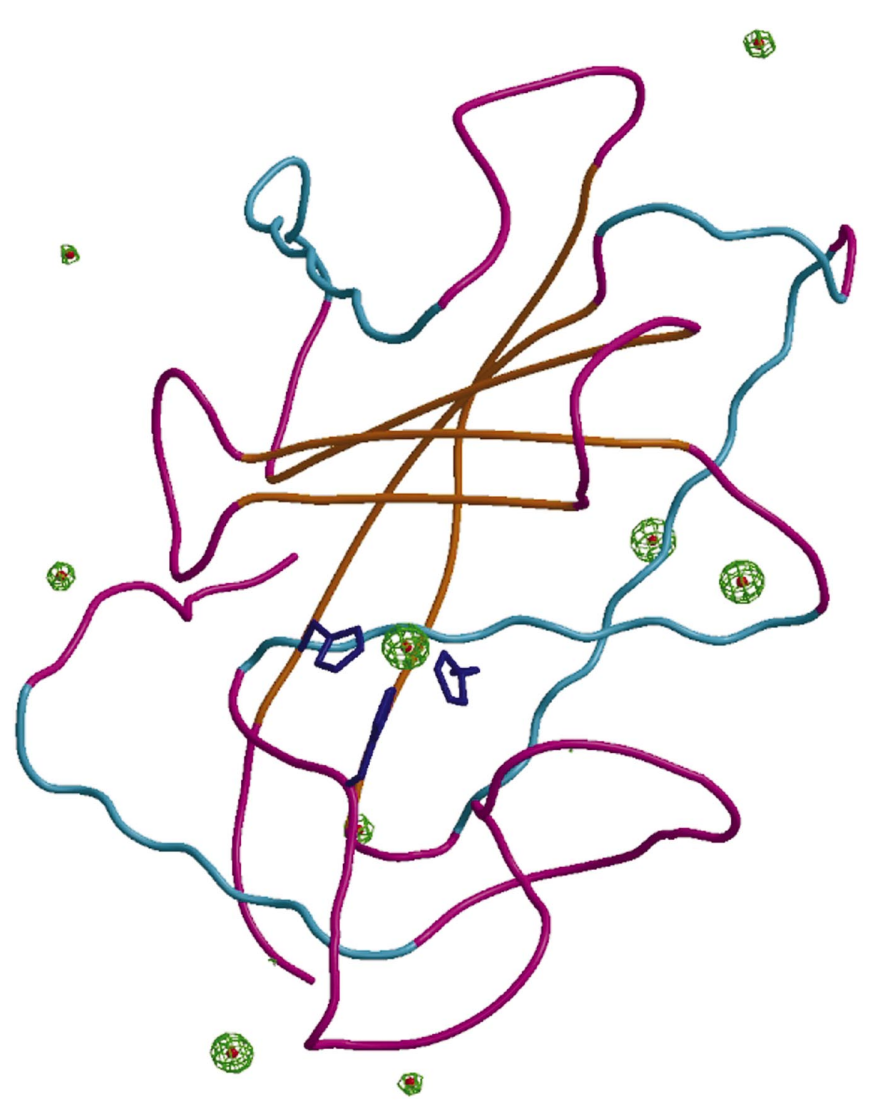

Figure 8

A $2 F_{o}-F_{c}$ map of the overall structure of TM-3 contoured at the $10.2 \sigma$ level. The electron-density map is drawn as green cages. Orientation is the same as in Fig. 2. Various secondary elements, referring to Fig. 2, are also coloured. The figure was prepared using BOBSCRIPT.

This work is supported in part by grants from Academia Sinica and the National Science Council (NSC 89-2311-B-001190 to S-HC), Taipei, Taiwan. We thank Dr Hideaki Moriyama of SPring-8 (Hyogo, Japan) and Dr Shun-Hsin Chen of the Institute of Molecular Biology at Academia Sinica (Taipei, Taiwan) for assistance in X-ray data collection. We also thank Ms Aileen Chuang of the Institute of Chemistry at Academia Sinica for assistance in the operation of the atomic absorption spectrophotometer.

\section{References}

Barlaam, B., Bird, T. G., Lambert-Van Der Brempt, C., Campbell, D., Foster, S. J. \& Maciewicz, R. (1999). J. Med. Chem. 42, 4890-4908.

Black, R. A., Rauch, C. T., Kozlosky, C. J., Peschon, J. J., Slack, J. L., Wolfson, M. F., Castner, B. J., Stocking, K. L., Reddy, P., Srinivasan, S., Nelson, N., Boiani, N., Schooley, K. A., Gerhart, M., Davis, R., Fitzner, J. N., Johnson, R. S., Paxton, R. J., March, C. J. \& Cerretti, D. P. (1997). Nature (London), 385, 729-733.

Bode, W., Gomis-Rüth, F. X., Huber, R., Zwilling, R. \& Stocker, W. (1992). Nature (London), 358, 164-167.

Bode, W., Grams, F., Reinemer, P., Gomis-Rüth, F. X., Baumann, U., McKay, D. B. \& Stöcker, W. (1996). Adv. Exp. Med. Biol. 389, 1-11.
Brünger, A. T., Adams, P. D., Clore, G. M., Delano, W. L., Gros, P., Grosse-Kunstleve, R. W., Jiang, J. S., Kuszewski, J., Nilges, M., Pannu, N. S., Read, R. J., Rice, L. M., Simonson, T. \& Warren, G. L. (1998). Acta Cryst. D54, 905-921.

Bukrinsky, J. T., Bjerrum, M. J. \& Kadziola, A. (1998). Biochemistry, 37, 16555-16564.

Catanese, J. J. \& Kress, L. F. (1992). Biochemistry, 31, 410-418.

Cho, J. H., Kim, D. H., Lee, K. J., Kim, D. H. \& Choi, K. Y. (2001). Biochemistry, 40, 10197-10203.

Cirilli, M., Gallina, C., Gavuzzo, E., Giordano, C., Gomis-Rüth, F. X., Gorini, B., Kress, L. F., Mazza, F., Paradisi, M. P., Pochetti, G. \& Politi, V. (1997). FEBS Lett. 418, 319-322.

D'Alessio, S., Gallina, C., Gavuzzo, E., Giordano, C., Gorini, B., Mazza, F., Paradisi, M. P., Panini, G., Pochetti, G. \& Sella, A. (1999). Bioorg. Med. Chem. 7, 389-394.

Dugas, H. (1989). Bioorganic Chemistry: a Chemical Approach to Enzyme Action, edited by H. Dugas, pp. 400-408. New York: Springer-Verlag.

Engh, R. A. \& Huber, R. (1991). Acta Cryst. A47, 392-400.

Fox, J. W. \& Long, C. (1998). Enzymes from Snake Venom, edited by G. S. Bailey, pp. 151-178. Fort Collins: Alaken.

Francis, B. \& Kaiser, I. I. (1993). Toxicon, 31, 889-899.

Gill, S. C. \& von Hippel, P. H. (1989). Anal. Biochem. 182, 319326.

Gomis-Rüth, F. X., Kress, L. F., Kellermann, J., Mayr, I., Lee, X., Huber, R. \& Bode, W. (1994). J. Mol. Biol. 239, 513-544.

Gomis-Rüth, F. X., Meyer, E. F., Kress, L. F. \& Politi, V. (1998). Protein Sci. 7, 283-292.

Gong, W., Zhu, X., Liu, S., Teng, M. \& Niu, L. (1998). J. Mol. Biol. 283, 657-668.

Hori, T., Kumasaka, T., Yamamoto, M., Nonaka, T., Tanaka, N., Hashimoto, Y., Ueki, T. \& Takio, K. (2001). Acta Cryst. D57, 361368.

Huang, K. F., Chow, L. P. \& Chiou, S. H. (1999). Biochem. Biophys. Res. Commun. 263, 610-616.

Huang, K. F., Hung, C. C. \& Chiou, S. H. (1993). Biochem. Mol. Biol. Int. 31, 1041-1050.

Huang, K. F., Hung, C. C., Pan, F. M., Chow, L. P., Tsugita, A. \& Chiou, S. H. (1995). Biochem. Biophys. Res. Commun. 216, $223-$ 233.

Huang, K. F., Hung, C. C., Wu, S. H. \& Chiou, S. H. (1998). Biochem. Biophys. Res. Commun. 248, 562-568.

Jones, T. A., Zou, J. Y., Cowan, S. W. \& Kjeldgaard, M. (1991). Acta Cryst. A47, 110-119.

Kumasaka, T., Yamamoto, M., Moriyama, H., Tanaka, N., Sato, M., Katsube, Y., Yamakawa, Y., Omori-Satoh, T., Iwanaga, S. \& Ueki, T. (1996). J. Biochem. 119, 49-57.

Laskowski, R. A., MacArthur, M. W., Moss, D. S. \& Thornton, J. M. (1993). J. Appl. Cryst. 26, 283-291.

Lipscomb, W. N. (1974). Tetrahedron, 30, 1725-1732.

Lipscomb, W. N. \& Sträter, N. (1996). Chem. Rev. 96, 2375-2433.

Maskos, K., Fernandez-Catalan, C., Huber, R., Bourenkov, G. P., Bartunik, H., Ellestad, G. A., Reddy, P., Wolfson, M. F., Rauch, C. T., Castner, B. J., Davis, R., Clarke, H. R., Petersen, M., Fitzner, J. N., Cerretti, D. P., March, C. J., Paxton, R. J., Black, R. A. \& Bode, W. (1998). Proc. Natl Acad. Sci. USA, 95, 3408-3412.

Meisch, H. U. \& Schmitt, J. A. (1986). Environ. Health Perspect. 65, 29-32.

Mock, W. L. \& Yao, J. (1997). Biochemistry, 36, 4949-4958.

Moreland, L. W., Baumgartner, S. W., Schiff, M. H., Tindall, E. A., Fleischmann, R. M., Weaver, A. L., Ettlinger, R. E., Cohen, S., Koopman, W. J., Mohler, K., Widmer, M. B. \& Blosch, C. M. (1997). N. Engl. J. Med. 337, 141-147.

Moss, M. L. et al. (1997). Nature (London), 385, 733-736.

Nair, P. S. \& Robinson, W. E. (1999). Arch. Biochem. Biophys. 366, 814.

Navaza, J. (1994). Acta Cryst. A50, 157-163.

Otwinowski, Z. \& Minor, W. (1997). Methods Enzymol. 276, 307-326. 
Robeva, A., Politi, V., Shannon, J. D., Bjarnason, J. B. \& Fox, J. W. (1991). Biomed. Biochim. Acta, 50, 769-773.

Rooke, J., Pan, D., Xu, T. \& Rubin, G. M. (1996). Science, 273, $1227-$ 1231.

Shannon, J. D., Baramova, E. N., Bjarnasson, J. B. \& Fox, J. W. (1989). J. Biol. Chem. 264, 175-190.

Stams, T., Spurlino, J. C., Smith, D. L., Wahl, R. C., Ho, T. F., Qoronfleh, M. W., Banks, T. M. \& Rubin, B. (1994). Nature Struct. Biol. 1, 119-123.

Stöcker, W. \& Bode, W. (1995). Curr. Opin. Struct. Biol. 5, 383-390. Stöcker, W., Grams, F., Baumann, U., Reinemer, P., Gomis-Rüth, F. X., McKay, D. B. \& Bode, W. (1995). Protein Sci. 4, 823-840.

Takeya, H., Arakawa, M., Miyata, T., Iwanaga, S. \& Omori-Satoh, T. (1989). J. Biochem. 106, 151-157.
Takeya, H. \& Iwanaga, S. (1998). Enzymes from Snake Venom, edited by G. S. Bailey, pp. 11-38. Fort Collins: Alaken.

Tortorella, M. D. et al. (1999). Science, 284, 1664-1666.

Tsai, I. H., Wang, Y. M., Chiang, T. Y., Chen, Y. L. \& Huang, R. J. (2000). Eur. J. Biochem. 267, 1359-1367.

Vilcek, J. \& Lee, T. H. (1991). J. Biol. Chem. 266, 7313-7316.

Wolfsberg, T. G. \& White, J. M. (1996). Dev. Biol. 180, 389-401.

Xue, C. B. et al. (2001). J. Med. Chem. 44, 2636-2660.

Yamakawa, Y. \& Omori-Satoh, T. (1992). J. Biochem. 112, 583-589.

Yiallouros, I., Berkhoff, E. G. \& Stöcker, W. (2000). FEBS Lett. 484, 224-228.

Zhang, D., Botos, I., Gomis-Rüth, F. X., Doll, R., Blood, C., Njoroge, F. G., Fox, J. W., Bode, W. \& Meyer, E. F. (1994). Proc. Natl Acad. Sci. USA, 91, 8447-8451. 